九州大学学術情報リポジトリ

Kyushu University Institutional Repository

\title{
A BIRTH PROCESS WITH A RANDOM BIRTH RATE
}

Dayal, M

Department of Statistics and Applied Probability, University of California

Gani, J

School of Mathematical Sciences, Australian National University

Haynatzki, G

Department of Statistics and Applied Probability, University of California

Iyer, $S$

Department of Statistics and Applied Probability, University of California

他

https://doi.org/10.5109/13441

出版情報: Bulletin of informatics and cybernetics. 27 (1)，pp.23-30，1995-03. Research Association of Statistical Sciences

バージョン :

権利関係 : 


\title{
A BIRTH PROCESS WITH A RANDOM BIRTH RATE
}

\author{
By
}

\section{Dayal, J. Gani, G. Haynatzki, S. IyeR; and N. SchumaCher ${ }^{*}$}

\begin{abstract}
This note extends the results of the classical birth process to the case where the birth parameter is a two-state Markov chain. Partial differential equations for the two components $\phi_{1}(z, t)$ and $\phi_{2}(z, t)$ of the probability generating function of the process are derived, and Laplace transforms of these taken. Solutions are then found for $\phi_{1}(z, t)$ and $\phi_{2}(z, t)$ by standard methods of analysis.
\end{abstract}

\section{Introduction}

This note is concerned with a birth process in which the birth parameter $\{L(t) ; t \geq 0\}$ is itself stochastic and may take either of two values $L_{1}, L_{2}$. The classical birth process was discussed by McKendrick (1914) (see also Bartlett (1949) and Kendall (1949)). For simplicity, we shall assume that $\{L(t) ; t \geq 0\}$ is a two-state Markov chain in continuous time, with initial value $L(0)=L_{1}$. Let the infinitesimal transition probabilities of this process be given by

$$
\begin{aligned}
& P\left\{L(t+\delta t)=L_{2} \mid L(t)=L_{1}\right\}=\lambda_{1} \delta t+o(\delta t), \\
& P\left\{L(t+\delta t)=L_{1} \mid L(t)=L_{2}\right\}=\lambda_{2} \delta t+o(\delta t), \\
& P\left\{L(t+\delta t)=L_{1} \mid L(t)=L_{1}\right\}=1-\lambda_{1} \delta t-o(\delta t), \\
& P\left\{L(t+\delta t)=L_{2} \mid L(t)=L_{2}\right\}=1-\lambda_{2} \delta t-o(\delta t),
\end{aligned}
$$

and the probability of two or more events in $(t, t+\delta t)$ be $o(\delta t)$. Then we can readily derive the probabilities

$$
\begin{aligned}
& q_{1}(t)=P\left\{L(t)=L_{1} \mid L(0)=L_{1}\right\}=\frac{\lambda_{2}}{\lambda_{1}+\lambda_{2}}+\frac{\lambda_{1}}{\lambda_{1}+\lambda_{2}} e^{-\left(\lambda_{1}+\lambda_{2}\right) t} \\
& q_{2}(t)=P\left\{L(t)=L_{2} \mid L(0)=L_{1}\right\}=\frac{\lambda_{1}}{\lambda_{1}+\lambda_{2}}-\frac{\lambda_{1}}{\lambda_{1}+\lambda_{2}} e^{-\left(\lambda_{1}+\lambda_{2}\right) t}
\end{aligned}
$$

\footnotetext{
* Department of Statistics and Applied Probability, University of California, Santa Barbara, Ca, 931063110,U.S.A.

† School of Mathematical Sciences, Australian National University, Canberra, ACT 2601, Australia. e-mail: gani@orac.anu.edu.ac
} 
The Laplace transforms $\int_{0}^{\infty} e^{-s t} q_{i}(t) d t, i=1,2, \operatorname{Re}(s) \geq 0$, of these probabilities are

$$
\begin{aligned}
& \hat{q}_{1}(s)=\frac{\lambda_{2}}{\lambda_{1}+\lambda_{2}} \cdot \frac{1}{s}+\frac{\lambda_{1}}{\lambda_{1}+\lambda_{2}} \cdot \frac{1}{s+\lambda_{1}+\lambda_{2}}, \\
& \hat{q}_{2}(s)=\frac{\lambda_{1}}{\lambda_{1}+\lambda_{2}} \cdot \frac{1}{s}-\frac{\lambda_{1}}{\lambda_{1}+\lambda_{2}} \cdot \frac{1}{s+\lambda_{1}+\lambda_{2}} .
\end{aligned}
$$

We shall allow this two-state Markov chain to drive the birth process $\{X(t) ; t \geq 0\}$, and obtain a formal solution for its probability generating function (p.g.f.)

$$
\phi(z, t)=E\left(z^{X(t)}\right), \quad|z| \leq 1
$$

by solving a matrix partial differential equation.

General methods for analyzing such processes have been developed by Puri (1975, 1976), but his methods though powerful, are rather complicated. In this note, we attempt to give a simple example of how bivariate Markov chains in continuous time, namely $\{(L(t), X(t)) ; t \geq 0\}$, can be useful in obtaining relatively simple results for a stochastic process influenced by another stochastic process.

\section{The Kolmogorov Equations For $\{(L(t), X(t)) ; t \geq 0\}$}

We shall now assume that the process $\{(L(t), X(t)) ; t \geq 0\}$ in continuous time for $j \geq 1$ is subject to the following infinitesimal transition probabilities:

$$
\begin{aligned}
P\left\{L(t+\delta t)=L_{2}, X(t+\delta t)=j \mid L(t)=L_{1}, X(t)=j\right\} & =\lambda_{1} \delta t+o(\delta t), \\
P\left\{L(t+\delta t)=L_{1}, X(t+\delta t)=j \mid L(t)=L_{2}, X(t)=j\right\} & =\lambda_{2} \delta t+o(\delta t), \\
P\left\{L(t+\delta t)=L, X(t+\delta t)=j+1 \mid L(t)=L_{1}, X(t)=j\right\} & =L_{1} j \delta t+o(\delta t), \\
P\left\{L(t+\delta t)=L_{2}, X(t+\delta t)=j+1 \mid L(t)=L_{2}, X(t)=j\right\} & =L_{2} j \delta t+o(\delta t),
\end{aligned}
$$

The probability of no event occuring in $(t, t+\delta t)$ is given by the usual complementary probability, and the probability of more than one event is $o(\delta t)$.

By the usual methods for deriving the Kolmogorov equations, we find that for $j=1,2, \ldots$, the probabilities

$$
\begin{aligned}
& p_{1 j}(t)=P\left\{L(t)=L_{1}, X(t)=j \mid L(0)=L_{1}, X(0)=1\right\} \\
& p_{2 j}(t)=P\left\{L(t)=L_{2}, X(t)=j \mid L(0)=L_{1}, X(0)=1\right\}
\end{aligned}
$$

satisfy the differential equations

$$
\begin{aligned}
\frac{d p_{1 j}}{d t} & =L_{1}(j-1) p_{1, j-1}-\left(\lambda_{1}+j L_{1}\right) p_{1 j}+\lambda_{2} p_{2 j} \\
\frac{d p_{2 j}}{d t} & =L_{2}(j-1) p_{2, j-1}-\left(\lambda_{2}+j L_{2}\right) p_{2 j}+\lambda_{1} p_{1 j}
\end{aligned}
$$


where for convenience we define $p_{10}=p_{20}=0$ when $j=1$ in the above equations, and it is clear that $p_{11}(0)=1, p_{21}(0)=0$.

These equations may be written in matrix form, in terms of $P_{1}^{\prime}=\left(p_{11}, p_{12}, \ldots\right)$, $P_{2}^{\prime}=\left(p_{21}, p_{22}, \ldots\right)$, as

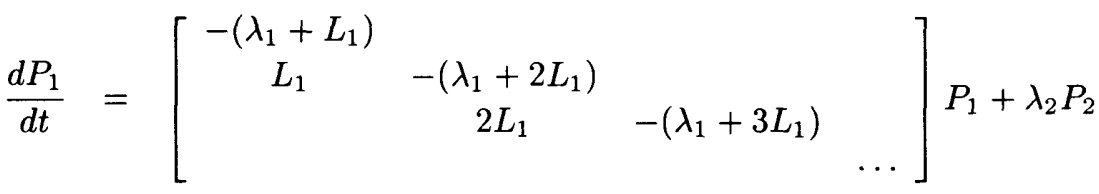

$$
\begin{aligned}
& =-A_{1} P_{1}+\lambda_{2} P_{2} \text {, }
\end{aligned}
$$

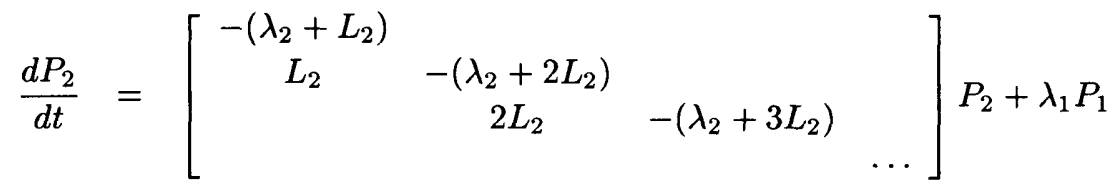

$$
\begin{aligned}
& =-A_{2} P_{2}+\lambda_{1} P_{1} \text {. }
\end{aligned}
$$

Note that the matrices $A_{1}, A_{2}$ are infinite, so that matrix methods cannot be used directly. If, however, the process is terminated when $X(t)=N$, say, the matrices will be finite (though slightly altered for the value $N$ ) so that the problem becomes one of a finite-state Markov chain.

In order to solve these equations, we consider the generating functions (g.f.)

$$
\begin{array}{ll}
\phi_{1}(z, t)=\sum_{j=1}^{\infty} z^{j} p_{1 j}(t), \\
\phi_{2}(z, t)=\sum_{j=1}^{\infty} z^{j} p_{2 j}(t), & |z| \leq 1 .
\end{array}
$$

Note that p.g.f. $\phi(z, t)$ of (1.3) is in effect $\phi=\phi_{1}+\phi_{2}$, where the latter are themselves not p.g.f.s. From (2.3) we can readily derive the partial differential equations

$$
\begin{aligned}
\frac{\partial \phi_{1}}{\partial t} & =-\lambda_{1} \phi_{1}+L_{1} z(z-1) \frac{\partial \phi_{1}}{\partial z}+\lambda_{2} \phi_{2}, \\
\frac{\partial \phi_{2}}{\partial t} & =-\lambda_{2} \phi_{2}+L_{2} z(z-1) \frac{\partial \phi_{2}}{\partial z}+\lambda_{1} \phi_{1},
\end{aligned}
$$

which need to be solved for the probabilities $p_{1 j}(t), p_{2 j}(t)$ to be found.

\section{Laplace Transforms and Method of Solution}

In order to simplify equations (2.3), let us first take Laplace transforms with respect to $t$ such that for $\operatorname{Re}(s) \geq 0$, 


$$
\begin{aligned}
& \hat{\phi}_{1}(z, s)=\int_{0}^{\infty} e^{-s t} \phi_{1}(z, t) d t \\
& \hat{\phi}_{2}(z, s)=\int_{0}^{\infty} e^{-s t} \phi_{2}(z, t) d t .
\end{aligned}
$$

Then, the equations (2.3) yield

$$
\begin{aligned}
s \hat{\phi}_{1}-z & =L_{1} z(z-1) \frac{\partial \hat{\phi}_{1}}{\partial z}-\lambda_{1} \hat{\phi}_{1}+\lambda_{2} \hat{\phi}_{2} \\
s \hat{\phi}_{2} & =L_{2} z(z-1) \frac{\partial \hat{\phi}_{2}}{\partial z}+\lambda_{1} \hat{\phi}_{1}-\lambda_{2} \hat{\phi}_{2} .
\end{aligned}
$$

We may rewrite these in matrix form as

$$
\frac{\partial}{\partial z}\left[\begin{array}{c}
\hat{\phi}_{1} \\
\hat{\phi}_{2}
\end{array}\right]=\frac{1}{z(z-1)}\left\{\left[\begin{array}{cc}
\frac{\lambda_{1}+s}{L_{1}} & -\frac{\lambda_{2}}{L_{1}} \\
-\frac{\lambda_{1}}{L_{2}} & \frac{\lambda_{2}+s}{L_{2}}
\end{array}\right]\left[\begin{array}{c}
\hat{\phi}_{1} \\
\hat{\phi}_{2}
\end{array}\right]-\left[\begin{array}{c}
z \\
\frac{z}{L_{1}} \\
0
\end{array}\right]\right\}
$$

or

$$
\frac{\partial}{\partial z} \hat{\boldsymbol{\phi}}=\frac{1}{z(z-1)}\{\mathbf{A} \hat{\boldsymbol{\phi}}-\mathbf{B} z\}
$$

where

$$
\mathbf{A}=\left[\begin{array}{cc}
\frac{\lambda_{1}+s}{L_{1}} & -\frac{\lambda_{2}}{L_{1}} \\
-\frac{\lambda_{1}}{L_{2}} & \frac{\lambda_{2}+s}{L_{2}}
\end{array}\right] \text { and } \mathbf{B}=\left[\begin{array}{c}
\frac{1}{L_{1}} \\
0
\end{array}\right]
$$

This form leads us to the method of solution, in which we transform the variable $z$ to $\zeta=\ln \frac{z}{1-z}$, so that we have $\hat{\phi}(\zeta, s)$ now a function of $\zeta$ and $s$ satisfying

$$
\frac{\partial}{\partial \zeta} \hat{\boldsymbol{\phi}} \cdot \frac{d \zeta}{d z}=\frac{1}{z(1-z)} \frac{\partial}{\partial \zeta} \hat{\boldsymbol{\phi}}=-\frac{1}{z(1-z)} \mathbf{A} \hat{\boldsymbol{\phi}}+\frac{1}{L_{1}(1-z)} \mathbf{E}
$$

where $\mathbf{E}^{\prime}=[1,0]$, or since $z=\frac{e^{\zeta}}{1+e^{\zeta}}$,

$$
\frac{\partial}{\partial \zeta} \hat{\boldsymbol{\phi}}+\mathbf{A} \hat{\boldsymbol{\phi}}=\frac{e^{\zeta}}{L_{1}\left(1+e^{\zeta}\right)} \mathbf{E} .
$$

To solve (3.4), we require the integrating factor $e^{\mathbf{A} \zeta}$, so that

$$
\frac{\partial}{\partial \zeta}\left(e^{\mathbf{A} \zeta} \hat{\boldsymbol{\phi}}\right)=e^{\mathbf{A} \zeta} \frac{\partial}{\partial \zeta} \hat{\boldsymbol{\phi}}+e^{\mathbf{A} \zeta} \mathbf{A} \hat{\boldsymbol{\phi}}=e^{\mathbf{A} \zeta} \frac{e^{\zeta}}{L_{1}\left(1+e^{\zeta}\right)} \mathbf{E} .
$$

Integrating (3.5) with respect to $\zeta$, we find

$$
e^{\mathbf{A} \zeta} \hat{\boldsymbol{\phi}}=\int e^{\mathbf{A} \zeta} \frac{e^{\zeta}}{L_{1}\left(1+e^{\zeta}\right)} \mathbf{E} d \zeta+\mathbf{F}(s)
$$


or, in terms of $z$,

$$
e^{\mathbf{A} \ln \left(\frac{z}{1-z}\right)} \hat{\phi}=\int_{0}^{z} e^{\mathbf{A} \ln \left(\frac{u}{1-u}\right)} \frac{\mathbf{E}}{L_{1}} \frac{d u}{(1-u)}+\mathbf{F}(s),
$$

where $\mathbf{F}^{\prime}(s)=\left[f_{1}(s), f_{2}(s)\right]$, these being unknown functions of $s$. Thus,

$$
\hat{\boldsymbol{\phi}}=e^{-\mathbf{A} \ln \left(\frac{z}{1-z}\right)} \int_{0}^{z} e^{\mathbf{A} \ln \left(\frac{u}{1-u}\right)} \frac{\mathbf{E}}{L_{1}} \frac{d u}{(1-u)}+e^{-\mathbf{A} \ln \left(\frac{z}{1-z}\right)} \mathbf{F}(s) .
$$

It now remains to work out the integral in (3.6).

\section{Solution of the Integral}

The form of $\mathbf{A}$ has been found to be

$$
\mathbf{A}=\left[\begin{array}{cc}
\frac{\lambda_{1}+s}{L_{1}} & -\frac{\lambda_{2}}{L_{1}} \\
-\frac{\lambda_{1}}{L_{2}} & \frac{\lambda_{2}+s}{L_{2}}
\end{array}\right]=\left[\begin{array}{cc}
a(s) & b \\
c & d(s)
\end{array}\right]
$$

Suppose we write this in the canonical form

$$
\mathbf{A}=\mathbf{B}^{-1} \mathbf{\Lambda} \mathbf{B}
$$

where $\boldsymbol{A}$ is the diagonal matrix

$$
\boldsymbol{\Lambda}=\left[\begin{array}{cc}
\rho_{1}(s) & 0 \\
0 & \rho_{2}(s)
\end{array}\right]
$$

with

$$
\begin{aligned}
& \rho_{1}(s)=\frac{a+d+\sqrt{(a-d)^{2}+4 b c}}{2} \\
& \rho_{2}(s)=\frac{a+d-\sqrt{(a-d)^{2}+4 b c}}{2}
\end{aligned}
$$

and

$$
\begin{aligned}
\mathbf{B} & =\left[\begin{array}{ll}
b_{11} & b_{12} \\
b_{21} & b_{22}
\end{array}\right]=\left[\begin{array}{ll}
c & -\left(a-\rho_{1}\right) \\
c & -\left(a-\rho_{2}\right)
\end{array}\right] \\
\mathbf{B}^{-1} & =\left[\begin{array}{ll}
b^{11} & b^{12} \\
b^{21} & b^{22}
\end{array}\right]=\frac{1}{c\left(\rho_{2}-\rho_{1}\right)}\left[\begin{array}{cc}
-\left(a-\rho_{2}\right) & \left(a-\rho_{1}\right) \\
-c & c
\end{array}\right] .
\end{aligned}
$$

Then 


$$
\begin{aligned}
e^{-\mathbf{A} \ln \left(\frac{z}{1-z}\right)} & =\mathbf{B}^{-1}\left[\begin{array}{cc}
e^{-\rho_{1} \ln \left(\frac{z}{1-z}\right)} & 0 \\
0 & e^{-\rho_{2} \ln \left(\frac{z}{1-z}\right)}
\end{array}\right] \mathbf{B} \\
& =\mathbf{B}^{-1}\left[\begin{array}{cc}
\left(\frac{1-z}{z}\right)^{\rho_{1}} & 0 \\
0 & \left(\frac{1-z}{z}\right)^{\rho_{2}}
\end{array}\right] \mathbf{B} .
\end{aligned}
$$

We now show that for some $s>0, \rho_{1}(s)$ and $\rho_{2}(s)$ are both positive. Writing out the values in (4.3) in detail, we have

$$
\begin{gathered}
\rho_{1,2}(s)=\frac{1}{2 L_{1} L_{2}}\left\{\lambda_{1} L_{2}+\lambda_{2} L_{1}+s\left(L_{1}+L_{2}\right)\right. \\
\left. \pm \sqrt{\left(\lambda_{1} L_{2}+\lambda_{2} L_{1}\right)^{2}+2 s\left(L_{1}+L_{2}\right)\left(\lambda_{1} L_{2}+\lambda_{2} L_{1}\right)+s^{2}\left(L_{1}+L_{2}\right)^{2}-4 s\left(s+\lambda_{1}+\lambda_{2}\right) L_{1} L_{2}}\right\} \\
=\frac{1}{2 L_{1} L_{2}}\left\{\lambda_{1} L_{2}+\lambda_{2} L_{1}+s\left(L_{1}+L_{2}\right) \pm \sqrt{\left[\lambda_{2} L_{1}-\lambda_{1} L_{2}+s\left(L_{1}-L_{2}\right)\right]^{2}+4 \lambda_{1} \lambda_{2} L_{1} L_{2}}\right\}
\end{gathered}
$$

where $\sqrt{\left[\lambda_{2} L_{1}-\lambda_{1} L_{2}+s\left(L_{1}-L_{2}\right)\right]^{2}+4 \lambda_{1} \lambda_{2} L_{1} L_{2}}$ is clearly positive and may be expressed as $\lambda_{2} L_{1}-\lambda_{1} L_{2}+\epsilon$, with $\epsilon>0$. Note that for all $s \geq 0$,

$$
\rho_{1}(s)=\frac{1}{2 L_{1} L_{2}}\left\{2 \lambda_{2} L_{1}+2 s L_{1}+\epsilon\right\}>0
$$

while

$$
\rho_{2}(s)=\frac{1}{2 L_{1} L_{2}}\left\{2 \lambda_{2} L_{1}+2 s L_{1}-\epsilon\right\}>0
$$

only for $2 L_{2}\left(s+\lambda_{1}\right)>\epsilon$, or $s>\frac{\epsilon}{2 L_{2}}-\lambda_{1}=s_{0}$. Hence both $\rho_{1}(s)$ and $\rho_{2}(s)$ will be positive for all $s>\max \left(0, s_{0}\right)=s_{1}$.

Consider, for $s>s_{1}$, the last component in (3.6),

$$
\begin{aligned}
& e^{-\mathbf{A} \ln \left(\frac{z}{1-z}\right)} \mathbf{F}(s)=\mathbf{B}^{-1}\left[\begin{array}{cc}
\left(\frac{1-z}{z}\right)^{\rho_{1}} & 0 \\
0 & \left(\frac{1-z}{z}\right)^{\rho_{2}}
\end{array}\right]\left[\begin{array}{ll}
b_{11} & b_{12} \\
b_{21} & b_{22}
\end{array}\right]\left[\begin{array}{l}
f_{1}(s) \\
f_{2}(s)
\end{array}\right] \\
& =\mathbf{B}^{-1}\left[\begin{array}{cc}
\left(\frac{1-z}{z}\right)^{\rho_{1}} & 0 \\
0 & \left(\frac{1-z}{z}\right)^{\rho_{2}}
\end{array}\right]\left[\begin{array}{l}
b_{11} f_{1}+b_{12} f_{2} \\
b_{21} f_{1}+b_{22} f_{2}
\end{array}\right] \\
& =\mathbf{B}^{-1}\left[\begin{array}{c}
\left(\frac{1-z}{z}\right)^{\rho_{1}}\left(b_{11} f_{1}+b_{12} f_{2}\right) \\
\left(\frac{1-z}{z}\right)^{\rho_{2}}\left(b_{21} f_{1}+b_{22} f_{2}\right)
\end{array}\right] \text {, }
\end{aligned}
$$

and let $z \rightarrow 0$. Then $\left(\frac{1-z}{z}\right)^{\rho_{1}},\left(\frac{1-z}{z}\right)^{\rho_{2}}$ will tend to $\infty$, and unless $f_{1}(s)=f_{2}(s)=0$, so will the elements of the final column matrix in (4.5). Since this cannot occur, it follows that $f_{1}(s)=f_{2}(s)=0$.

We now concentrate on the integral in (3.6), namely 


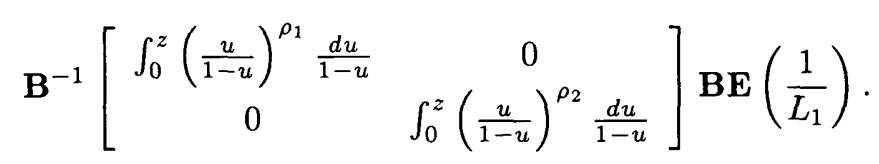

We first use the transformation $v=\frac{u}{1-u}$, such that $u=\frac{v}{1+v}, d u=\frac{d v}{(1+v)^{2}}$, to find that

$$
\int_{0}^{z}\left(\frac{u}{1-u}\right)^{\rho_{1}} \frac{d u}{1-u}=\int_{0}^{\frac{z}{1-z}} v^{\rho_{1}} \frac{d v}{1+v}
$$

Now integrating by parts, we obtain since $\int v^{\rho_{1}} \frac{d v}{1+v}=\frac{v^{\rho_{1}+1}}{\rho_{1}+1} \cdot \frac{1}{1+v}+\int \frac{v^{\rho_{1}+1}}{\rho_{1}+1} \cdot \frac{d v}{(1+v)^{2}}$, by repeated integration,

$$
\begin{aligned}
\int_{0} \frac{z}{1-z} v^{\rho_{1}} \frac{d v}{1+v}= & \left(\frac{z}{1-z}\right)^{\rho_{1}+1} \frac{(1-z)}{\rho_{1}+1}+\left(\frac{z}{1-z}\right)^{\rho_{1}+2} \frac{(1-z)^{2}}{\left(\rho_{1}+1\right)\left(\rho_{1}+2\right)}+ \\
& +2\left(\frac{z}{1-z}\right)^{\rho_{1}+3} \frac{(1-z)^{3}}{\left(\rho_{1}+1\right)\left(\rho_{1}+2\right)\left(\rho_{1}+3\right)} v+\cdots \\
& +\left(\frac{z}{1-z}\right)^{\rho_{1}} j ! z^{j+1} \frac{\Gamma\left(\rho_{1}+1\right)}{\Gamma\left(\rho_{1}+j+2\right)}+\cdots \\
= & \left(\frac{z}{1-z}\right)^{\rho_{1}} \sum_{j=0}^{\infty} j ! \frac{\Gamma\left(\rho_{1}+1\right)}{\Gamma\left(\rho_{1}+j+2\right)} z^{j+1}
\end{aligned}
$$

with a similar result holding for $\int_{0}^{z}\left(\frac{u}{1-u}\right)^{\rho_{2}} \frac{d u}{1-u}$. Hence, from (3.6)

$$
\begin{gathered}
\hat{\phi}(z, s) \\
=\mathbf{B}^{-1}\left[\begin{array}{cc}
\left(\frac{1-z}{z}\right)^{\rho_{1}} & 0 \\
0 & \left(\frac{1-z}{z}\right)^{\rho_{2}}
\end{array}\right] \mathbf{B} \times \\
\mathbf{B}^{-1}\left[\begin{array}{cc}
\left(\frac{z}{1-z}\right)^{\rho_{1}} \sum_{j=0}^{\infty} j ! \frac{\Gamma\left(\rho_{1}+1\right)}{\Gamma\left(\rho_{1}+j+2\right)} z^{j+1} & 0 \\
0 & \left(\frac{z}{1-z}\right)^{\rho_{2}} \sum_{j=0}^{\infty} j ! \frac{\Gamma\left(\rho_{2}+1\right)}{\Gamma\left(\rho_{2}+j+2\right)} z^{j+1}
\end{array}\right]\left[\begin{array}{c}
\frac{b_{11}}{L_{1}} \\
\frac{b_{21}}{L_{1}}
\end{array}\right] \\
=\left[\begin{array}{cc}
b^{11} & b^{12} \\
b^{21} & b^{22}
\end{array}\right]\left[\begin{array}{c}
\frac{b_{11}}{L_{1}} \sum_{j=0}^{\infty} j ! \frac{\Gamma\left(\rho_{1}+1\right)}{\Gamma\left(\rho_{1}+j+2\right)} z^{j+1} \\
\frac{b_{21}}{L_{1}} \sum_{j=0}^{\infty} j ! \frac{\Gamma\left(\rho_{2}+1\right)}{\Gamma\left(\rho_{2}+j+2\right)} z^{j+1}
\end{array}\right] \\
=\left[\begin{array}{c}
\frac{1}{L_{1}} \sum_{j=0}^{\infty} z^{j+1} j !\left(b^{11} b_{11} \frac{\Gamma\left(\rho_{1}+1\right)}{\Gamma\left(\rho_{1}+j+2\right)}+b^{12} b_{21} \frac{\Gamma\left(\rho_{2}+1\right)}{\Gamma\left(\rho_{2}+j+2\right)}\right) \\
\frac{1}{L_{1}} \sum_{j=0}^{\infty} z^{j+1} j !\left(b^{21} b_{11} \frac{\Gamma\left(\rho_{1}+1\right)}{\Gamma\left(\rho_{1}+j+2\right)}+b^{22} b_{21} \frac{\Gamma\left(\rho_{2}+1\right)}{\Gamma\left(\rho_{2}+j+2\right)}\right)
\end{array}\right]
\end{gathered}
$$

where the elements $b_{11}, b_{21}, b^{11}, b^{12}, b^{21}, b^{22}$ are known from (4.4).

This is effectively the formal solution of the problem in terms of Laplace transforms, involving the roots $\rho_{1}, \rho_{2}$. For,

$$
b^{11} b_{11}=\frac{\left(\rho_{2}-a\right)}{\left(\rho_{2}-\rho_{1}\right)}, \quad b^{12} b_{21}=\frac{\left(a-\rho_{1}\right)}{\left(\rho_{2}-\rho_{1}\right)},
$$




$$
b^{21} b_{11}=\frac{-c}{\left(\rho_{2}-\rho_{1}\right)}, \quad b^{22} b_{21}=\frac{c}{\left(\rho_{2}-\rho_{1}\right)},
$$

which also include the complicated square root of (4.4). It is interesting to note how the randomness of the birth parameter $\{L(t) ; t \geq 0\}$ has complicated a relatively simple process; nevertheless it is rewarding to find that one can obtain an explicit solution to this problem.

\section{Acknowledgment}

The second author worked on this paper with the support of NIH Grant R01AI29426.

\section{References}

[1] Bartlett, M.S. (1949) Some evolutionary stochastic processes. J. R. Statist. Soc. B 11, 211-229.

[2] Kendall, D.G. (1949) Stochastic processes and population growth. J. R. Statist. Soc. $B$ 11, 230-264.

[3] McKendrick, A.G. (1914) Studies on the theory of continuous probabilities, with special reference to its bearing on natural phenomena of a progressive nature. Proc. London Math. Soc. 13, 401-416.

[4] Puri, P.S. (1975) A linear birth and death process under the influence of another process. J. Appl. Prob. 12, 1-17.

[5] Puri, P.S. (1976) A stochastic process under the influence of another arising in the theory of epidemics. In Statistical Inference and Related Topics, Vol. 2, ed. M.L.Puri,235-255. Academic Press, New York.

Received December 26, 1994

Revised January 29, 1995 